\title{
THE ROLES OF VILLAGE HEALTH VOLUNTEERS: COVID-19 PREVENTION AND CONTROL IN THAILAND
}

\author{
Phudit Tejativaddhanal, Wichukorn Suriyawongpaisal', Vijj Kasemsup2, Thunwadee \\ Suksaroj ${ }^{1}$
}

1. ASEAN Institute for Health Development, Mahidol University, Thailand

2. Department of Family Medicine, Faculty of Medicine, Ramathibodi Hospital, Thailand and ASEAN Institute for Health Development, Mahidol University, Thailand

Correspondence: wichukorn1973@gmail.com

\begin{abstract}
Thailand has effectively responded to the COVID-19 pandemic with effective focus on primary healthcare and multi-sectoral collaboration with effective and open communication of powerful health messages. This article reviewed the central role of village health volunteers and their significant contribution to the disease control. The lessons from recent experiences need to be further evaluated to consolidate the lessons learned in anticipation of meeting continuing and future challenges.
\end{abstract}

\section{KEYWORDS}

COVID-19, management, prevention and control, Thailand, village health volunteers

\section{INTRODUCTION}

Since December 2019, coronavirus disease 2019, or COVID19 in short, has been a global challenge. Currently, there are 15,785,641 confirmed cases with an increase of 200,625 new cases and 640,016 deaths globally.[1] Surprisingly, countries in America like USA and Canada and European countries like UK and Italy recognized for their state of the art healthcare and use of screening and vaccination, have not done as well as some Asian countries like Thailand.[1] For example, while most of the hospitals in UK provide preventive care, the affluent appear to have better access to the service. One explanation to this issue is increasing privatization of health service and lower subsidization from the government, thus it increases health expenditure with low financial protection across the population. [2] On the other hand, the strong foundation of primary healthcare in Thailand complement the limited resources in health facilities in halting COVID-19.

According to the Declaration of Astana 2018, the success of primary health care depends on multi-sectoral policy and actions, empowered people and communities, together with primary care and the essential public health function.[3] The success in implementing primary health care not only mitigates the magnitude of the pandemic, but also reduce treatment costs for patients who need medical attention while promoting productivity of individuals who survived the pandemic.

Up till 27th July 2020, Thailand has 3,295 confirmed cases with 3,111 discharged cases and 58 deaths. [4] Nonetheless, with a strong foundation of a primary healthcare system, Thailand is able to control the disease within three months after country lockdown in March 2020 with the report of 'zero' new domestic cases for 48 consecutive days since June 2020.[5]

Among the key success factors of primary healthcare in Thailand is proactive collaboration between health and non-health sectors at the local level that helps infected individuals and their community members to live under the 'new normal' norm including social distancing, strictly using hygienic means to reduce infection, and so on. The patterns of collaboration can be divided into two 
categories: passive services and proactive services. Passive services include detection, screening, treatment, and follow-up on patients whereas active services cover health promotion activities. Each collaborative arm has been used simultaneously to decrease the burden of COVID-19 at the local level. However, some limitations in passive services hinder their effectiveness to tackle the disease at population scale. For example, setting up equipment and/or field health facilities for local quarantine require a large investment in order to support new and existing patients who need medical attention. As a result, the Ministry of Public Health (MOPH) and local authorities turn towards proactive services to promote the health of local individuals.[6]

However, delivery of proactive services can be troublesome at the local level due to limited resources, health personnel in particular, and trust-based communication to encourage health promotion for the locals. In this scenario, Thai village health volunteers (VHVs) play an important role in empowering people and their community to fend off the disease while assisting the health sector in reducing the burden of hospitalization of the patients. This article will describe the roles and the contribution of VHVs in addressing COVID-19 crisis at local scale.

\section{BACKGROUND OF VILLAGE HEALTH VOLUNTEERS}

The Thai government ratified the World Health Assembly Resolution on Primary Health Care in 1978 to expedite coverage of health care services at the local level. After adoption of the resolution, village health communicators (VHCS), whose main roles were to provide health communication, were formed along with VHVs. However, drastic improvement in primary health care and relevant management system ushered changes in roles of the $\mathrm{VHCs}$ and, in 1995, VHCs were integrated into VHVs with expanded roles relevant to supporting health professionals and facilitating collaboration between health and nonhealth sectors at local level.[7] In addition, the Centre for Community Primary Healthcare was formed at local in 2000 to enhance coordination of VHVs in their community. [6,7] The centre in each district offers basic health services provided by VHVs as well as provides a public space for improving local engagement in public health activities.[7]
The Thai government, particularly in the health sector, prioritises the importance of VHVs in empowering local individuals and communities to become resilient in respect to health issues. Training and retraining of VHVs were offered by the Ministry of Public Health (MOPH) to increase VHV's competencies for better collaboration with other health professionals in their local setting. Those competencies include screening, surveillance, follow-up and educating individuals to become better at taking care of their own health. Additional to $\mathrm{MOPH}$, programs or projects supported by Sub-district Health Fund [a subdistrict funding source for operationalizing health-related projects or programs at sub-district level] of National Health Security Office (NHSO) promotes capacity building on health promotion and disease prevention of VHVs.[7]

Aside from the support of health sector, district health boards (DHBs), a novel local structure supporting the promotion of health and well-being, is another supporting mechanism enabling VHV's contribution to policy implementation.[7] The DHB comprises of district level representatives from health and non-health sector with the District Chief as the chairperson of each DHB and the head of District Public Health Office as the secretariat. Some DHBs have Sub-district Health Boards (SHBs), a sub-structure of DHB, which facilitate district policy translation and implementation at sub-district level. VHVs involved in most SHBs as a committee member from health sector. Since VHVs were elected by their local communities, some health-related problems which might have been undetected by the local government were properly addressed. As a result, VHVs are essential contributors to DHB and local health system.[7]

\section{ROLES OF VILLAGE HEALTH VOLUNTEERS AGAINST COVID-19}

In Thailand, VHVs' performance to assist both government, health professionals, and local individuals was one of key factors in mitigating the spread of COVID-19. During the pandemic, VHVs' remarkable performance includes $[6,8,9,10]$,

1. Empowering people and community by promoting self-care and capacity of individuals to care for others through education to individuals in the community on prevention against COVID-19 (e.g., wearing masks, washing hands, and social distancing)

2. Supporting local surveillance system by assisting field epidemiological processes (e.g., proactive screening, 
timely report of early detected cases, follow-up on treated individuals, etc.) as well as assisting health professionals and individuals for local quarantine process

3. Reducing burden in hospitalization for COVID and non-COVID patients including forming a 'Grab Drug' network to help chronic patients acquire their medication without visiting a local hospital.

4. Help local community in managing Sub-district Health Fund to mobilize resources for COVID-19 prevention and control

Since January 2020, VHVs have demonstrated their roles in prevention of COVID-19 spreading. Their main responsibility included screening individuals in each household with particular focus on those who travelled back from foreign countries or high-risk areas at national level. The VHVs then contacted Sub-district Health Promotion Hospital in their working areas for referral of cases needing medical attention. Also, VHs provided emotional support for individuals to mitigate mental impact from the outbreak in their living areas and provided logistic service to improve access to medications for individuals with chronic conditions. $[8,9]$

Door-to-door assistance and close kinship between VHVs and local individuals promote compliance to national guidelines on prevention against and self-care for COVID19 including hand washing, applying hand sanitizers, hygienic mask wearing, and social distancing.[10] Unlike service delivery provided by health professionals at local health facilities, the door-to-door service reduce duration and distance gaps making it easier for performing early detection in remote areas. Also, the use of online communication platforms like LINE facilitate communication among the VHVs, local individuals, and health professionals which increase chance of referral for treatment at early sign of symptoms and online surveillance for local-quarantined individuals.[6]

Support from local authorities like DHB and collaboration at national level also enhance VHV efforts in COVID-19 prevention and control. The disease become the top policy priority in every district. As a result, resources including local funds were directed towards disease reduction. The Subdistrict Health Fund is also utilized to support capacity building for surveillance and health promotion for VHVs.[10] Additionally, a Thai telecommunication company collaborates with $\mathrm{MOPH}$ in developing an online application to promote a local surveillance system.[11]

\section{DISCUSSION}

VHVs and relevant supporting systems for primary healthcare in Thailand is crucial to ameliorate COVID-19 pandemic and future outbreaks, particularly in empowering individuals and communities to become resilient on health issues.[12] Without VHVs, the Thai health system may be overburden by increasing treatment costs and number of patients and, in the long run, it will result in a crippled national economy and welfare.

In March 2020 [12], an Emergency Decree was announced to contain the spread of the disease. Regulations according to the decree restrict travelling across provinces and setting up screening stations along provincial borders. However, before official announcement, most of Thai people started travelling to visit their family during Songkran Festival, a new-year celebration according to the Thai calendar. As a result, identification of infected patients was difficult. In addition, facts and rumours related to COVID-19 were mixed, thus making it even harder for local people to correctly comply with official guidelines.

However, VHVs who were stationed across different areas in each province were quick to respond to the pandemic and collaborate with the District Health Board to assist both health and non-health actors to control the disease. Since most VHVs grew up in their hometown and knew most of their community members, this helps them gaining trust from community members including those travelling back from other provinces. Hence, cooperation for screening and compliance with government guidelines for COVID-19 was mostly successful. This reinforces that 'trust' between the communities and health providers is essential in the time of pandemic, especially for addressing the issue in low-resource setting.[13]

However, there is room for improvement to optimize functionality of VHVs. First of all, the success of control of the disease is limited to some districts, if not provinces. This is due to the lack of systemic communication which can maximize efficiency of the communication across different provinces to share and customize good practices in

1 The innovation was adapted from 'Grab Bike', a food delivery service, and has been operated by Thai VHVs to transport drugs from local hospitals to individuals at home. $[7,8]$ 
COVID-19 prevention and control. Taiwan, as one example, demonstrated effective communication system to inform local individuals to effectively protect themselves against COVID-19 (e.g., when and where to wear masks and effective handwashing). Also, the communication system encouraged coordination across government sectors to mitigate the loss of opportunities and income during the outbreak while regulating activities that may affect disease control (e.g., hoarding masks, falsified information on the disease, failure to control immigration).[14,15] Secondly, VHVs may prove to be an effective mechanism in non-urban areas since the bond between community members and local VHVs is tighter than their urban counterparts. As a result, should the disease reemerge in the future, operationalizing of health volunteers requires customization for urban context.

\section{CONCLUSION}

Performance of Thai VHVs during COVID-19 pandemic contributes to the success of the disease prevention and control under the concept of primary healthcare as described in the Declaration of Astana 2018. Trust-based value in primary healthcare helps individuals living in remote areas reach essential services. However, capacity building for $\mathrm{VHVs}$ for using advance technology for communication can optimize timely healthcare delivery to avert future epidemic. Also, understanding relationship between individuals and healthcare providers in urban setting can unlock more opportunities to mitigate the magnitude of epidemics in densely populated areas.

\section{LIMITATIONS}

The article reflects commentary based on the analysis of current management practice during the pandemic. Subsequent evaluation and research into the longer-term outcomes of this approach may further improve the effectiveness of $\mathrm{VHV}$ mechanism.

\section{References}

1. World Health Organization (WHO). WHO coronavirus disease (covid-19) dashboard. Available: <https://covid 19.who.int/> (Accessed 27/07/20)

2. Baeten R, Spasova S, Vanhercke B, Coster S. Inequalities in access to healthcare a study of national policies. Brussels: European Commission; 2018.

3. WHO Communications materials global conference on primary health care. Available:

<https://www.who.int/primary-health/conferencephc/communications-materials> (Accessed 13/07/20)

4. Department of Disease Control (DDC). Covid-19 situation in Thailand (in Thai). Available: <https://ddc.moph.go.th/viralpneumonia/> (Accessed 27//07/2020)

5. Health Focus (Hfocus). Thailand is free from covid-19 new cases for 48 consecutive days (in Thai).

Available:

<https://www.hfocus.org/content/2020/07/19742>

(Accessed 13/07/20)

6. Thaipost. "Vhv" the frontline against covid (in Thai). Available:

<https://www.thaipost.net/main/detail/64503> (Accessed 13/07/20)

7. Ministry of Public Health. Thailand health profile 20162017 (in Thai). Nonthaburi: Ministry of Public Health; 2017.

8. ThaiPBS. Vhv to innovate "grab drug" for hospital-home drug delivery during COVID-19 pandemic (in Thai). Available: <https://news.thaipbs.or.th/content/291608> (Accessd $13 / 07 / 20)$

9. Hfocus. Thai vhvs deliver drugs to chronic patients at home to reduce crowding in Tah chang hospital, singburi province (in Thai). Available: <https://www.hfocus.org/content/2020/05/19383> (Accessed 13/07/20)

10. National Health Security Office (NHSO). The program for building health literacy for village health volunteers (vhv) for community caring (in Thai). Available: <https://localfund.happynetwork.org/project/5742> (Accessed 13/07/20)

11. Matemate. Ais to collaborate with moph in equiping vhv and health professionals to fight against covid-19 second wave (in Thai). Available: 
<https://brandinside.asia/ais-5g-fight-covid19/>

(Accessed 13/07/20)

12. BBC Thai. Coronavirus: Thai government to declare an emergency decree against covid-19 by this Thursday (in Thai). Available:

<https://www.bbc.com/thai/thailand-52014435>

(Accessed 27/07/2020)

13. Munonge J. Community and communication: fighting covid-19 in DRC. Available:

<https://mailchi.mp/612d4adc536f/what-werereading-4137310?e=8f54b0273e> (Accessed 13/07/20)

14. Wang CJ, Ng CY, Brook RH. Response to covid-19 in Taiwan: big data analytics, new technology, and proactive testing. JAMA 2020.

15. Emanuel EJ, Cathy Z, Glickman A. Learning from Taiwan about responding to covid-19 - and using electronic health records. Available: <https://www.statnews.com/2020/06/30/taiwanlessons-fighting-covid-19-using-electronic-healthrecords/> (Accessed 13/07/20) 